\title{
Migración de Finos en el Reservorio Hollín: Diagnóstico, Evaluación y Prácticas de Estimulación desde el Laboratorio hasta la Implementación en Campo
}

\author{
Ruilova, Mayra ${ }^{1, *}$ iD ; Izurieta, Álvaro² \\ ${ }^{1}$ Universidad Central del Ecuador, Facultad de Ingeniería en Geología, Minas, Petróleos y Ambiental, Quito, Ecuador. \\ ${ }^{2}$ Halliburton, Quito, Ecuador
}

\begin{abstract}
Resumen: El presente estudio muestra las diferentes pruebas de laboratorio utilizadas para reconocer los mecanismos de daño de formación y enlazar el comportamiento de producción asociado con la arenisca Hollín de la Cuenca Oriente. Se sugieren distintos tratamientos para mejorar la productividad y expandir la vida productiva del pozo. El análisis comienza con un diagnóstico con base en los históricos de producción del campo de estudio, definiendo la migración de finos como mecanismo de daño principal, esta hipótesis es luego verificada mediante Difracción de rayos X que nos mostraron la presencia de caolinita e ilita en los núcleos estudiados. Una vez identificado el mecanismo de daño del reservorio Hollín, se procedió a recomendar fluidos amigables con la formación, así como tratamientos de estimulación matricial con base en las pruebas de retorno de permeabilidad para fluidos de control, sistemas ácidos, estabilización de finos, y modificadores de permeabilidad relativa. Además, se propone fractura hidráulica en combinación con los tratamientos antes descritos como alternativa para mejorar y extender la vida productiva de los pozos.
\end{abstract}

Palabras clave: Retorno de permeabilidad, XRD, Estimulación Acida, Mecanismo de Daño, RPM, Fractura.

\section{Fines Migration in the Hollín Sandstone: Diagnosis, Evaluation and Stimulation Practices from the Laboratory to Field Implementation}

\begin{abstract}
This study shows laboratory tests used to recognize formation damage mechanisms and corresponding production behavior for the Hollin sandstone located in the Oriente Basin. Different stimulation treatments are suggested to improve productivity and expand the productive life of the well. The analysis begins with diagnosis based on historical production data where fines migration is hypnotized as a main formation damage mechanism, which is verified by the presence of kaolinite and illite in core data obtained from X-ray Diffraction tests. Once the main damage mechanism is identified, different treatments are recommended on regained permeability tests for completion brines, acid blends, fines stabilization, and relative permeability modifiers. Furthermore, hydraulic fracturing in combination with treatments described previously is proposed as an alternative to improve and extend the productive life of the well.
\end{abstract}

Keywords: Regained Permeability, XRD, Acid Stimulation, Damage Mechanism, RPM, Fracture.

\section{INTRODUCCIÓN}

Las operaciones de producción implican la interacción de diferentes tipos de fluidos con la roca reservorio. Al ingresar un fluido a la formación, este interactúa con el fluido original del yacimiento y la formación, ocasionando el desprendimiento de arcillas de migración como caolinita que tapona las gargantas porales. Este fenómeno se manifiesta como daño formación resultado en declinación de la producción esperada del pozo.

En los últimos años, se han desarrollado nuevas tecnologías que han ayudado a una mejor selección de fluidos orientados a mejorar y extender la vida productiva de los pozos como: fluidos de control, sistemas ácidos, fluidos de fractura y control de agua, sin embargo, estos fluidos pueden reaccionar de una manera diferente dependiendo las características petrofísicas y mineralógicas de la roca, presiones y características del crudo y agua producidos.

Uno de los principales mecanismos de daño de formación en los reservorios de la cuenca Oriente es la migración de finos, el cual se visualiza a lo largo de la producción, ya que existe una disminución de producción significativa al incrementar el corte de agua. La selección de fluidos adecuados a ser usados en el reservorio Hollín permitirá recuperar la productividad de 
la arena y disminuir el costo de producción por barril de la empresa operadora, y de esta manera incrementar los ingresos y rentabilidad de los proyectos petroleros.

\section{OBJETIVOS}

- Analizar los mecanismos de daño más frecuentes a través de pruebas de retorno de permeabilidad para la Arena Hollín en un campo petrolero del Oriente Ecuatoriano.

- Identificar las diferencias mineralógicas de los distintos núcleos para el reservorio Hollín.

- Determinar el mecanismo de daño que afecta a la Arena Hollín.

- Evaluar el retorno de permeabilidad en fluidos de control, sistemas ácidos, y control de agua específicos.

- Identificar estrategias para la prevención y / o reducción del daño potencial de la formación de pozos productores del reservorio Hollín.

\section{METODOLOGÍA}

\subsection{Diagnóstico}

Se realizó un diagnóstico con base en los diferentes casos históricos de producción del campo de estudio, observando el comportamiento de la producción de fluido en el tiempo. Además, se estudió los registros litológicos de los pozos que permitieron conocer mineralogía del reservorio, a través de estos primeros datos se observó un posible problema en la producción del reservorio Hollín.

Es importante destacar que el tipo de fluido de estimulación utilizado es un fluido ácido, el cual consta de 3 componentes importantes: fluido de control o prelavado, fluido de estimulación y un fluido de desplazamiento.

Cloruro de Amonio: Proporciona suficiente intercambio iónico y mantiene la concentración de sal para evitar que la arcilla se hinche antes y después del tratamiento ácido evitando el intercambio iónico.

Ácido Clorhídrico: Reacciona con las formaciones de carbonato y se utiliza como pre-flujo ácido en el tratamiento de formaciones de arenisca. El HCL puede servir como ácido básico para la eliminación de escala

Ácido Acético: este ácido es más débil que el ácido clorhídrico y presenta menos problemas de corrosión que el HCL; lo que significa que sus iones de hidrógeno se liberan más lentamente que el HCL con la mayoría de los materiales que se encuentran en los pozos. Debido a su bajo grado de corrosividad, los ácidos orgánicos pueden usarse a temperaturas más altas para tratamientos de acidificación. El ácido acético es el único ácido que no daña la tubería cromada usada en campos con alto contenido de $\mathrm{CO}_{2}$.

Bifluoruro de Amonio: Reacciona con el HCL para formar ácido fluorhídrico, HF. El HF disuelve las arcillas de migración como la caolinita.
Aditivo de control de arcillas: Controla la migración e hinchamiento de arcillas. Este se adsorbe en las gargantas porales evitando el contacto de las arcillas (finos de migración) con fluidos que provoquen intercambio iónico y posterior migración de finos.

Inhibidor de Corrosión: Limita la corrosión que pueda generar el tratamiento acido a los tubulares y equipos instalados.

Surfactante: Tiene la función de ayudar a la prevención de emulsiones y retorno de los fluidos inyectados una vez que el pozo entra en producción.

\section{Caso 1}

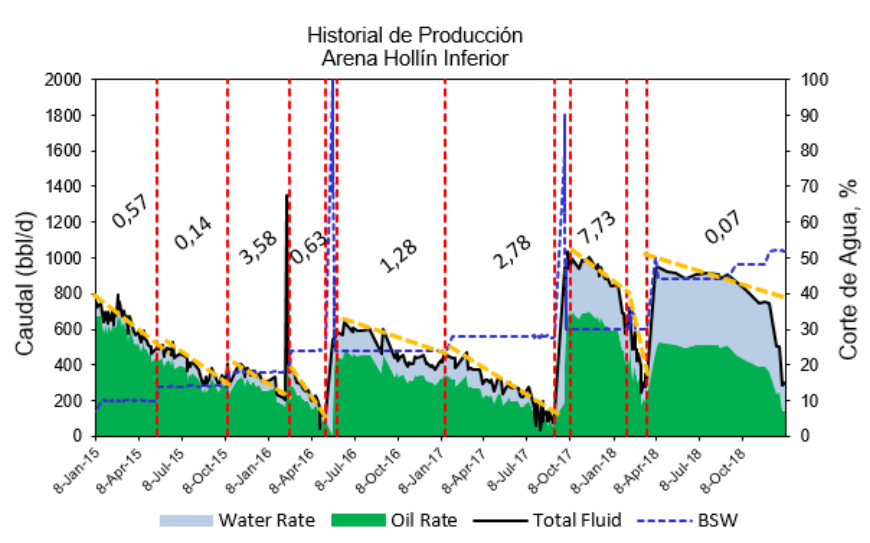

Figura 1. Caso 1 de Producción Campo de estudio

En este caso, se observa que conforme la producción de agua va incrementando, la producción de petróleo disminuye $\mathrm{m}=1.76,0.57$ y 0.14 (donde $\mathrm{m}=$ pendiente). La disminución de la producción inicial puede deberse a dos fenómenos: el primero debido al cambio en la permeabilidad relativa por el incremento de la saturación de agua, por presencia del acuífero constante en el reservorio Hollín (Baby et al, 2014), y el segundo debido a que existe una interacción entre el reservorio y el agua, lo cual provoca el comienzo de la migración de finos debido al intercambio iónico de presente en el reservorio y el agua que intruye como parte del mecanismo de producción natural del reservorio. (Civan, 2000), Figura 1.

En este caso de estudio, se considera al segundo escenario como válido debido a la respuesta de producción incremental posterior a la estimulación ácida, y dado que se realizan varias estimulaciones en la vida del pozo con respuesta positiva, es por eso por lo que se considera este efecto como el de mayor relevancia, sin que el primero sea descartado completamente.

El 8 de mayo del 2016, se realiza una primera rehabilitación de pozo en el cual se utilizó el diseño de fluido I. 
Tabla 1. Composición del fluido I

\begin{tabular}{cc}
\hline Químico & \% Volumen \\
\hline Bifluoruro de Amonio & $20 \%$ \\
$\mathrm{HCl}$ & $43,8 \%$ \\
Cloruro de Amonio & $43,8 \%$ \\
Inhibidor de corrosión & $0.4 \%$ \\
Surfactante & $0.2 \%$ \\
Composición del fluido II y III \\
\hline Químico & $\mathbf{\%}$ Volumen \\
\hline Bifluoruro de Amonio & $20 \%$ \\
HCl & $43,8 \%$ \\
Cloruro de Amonio & $7,7 \%$ \\
Ácido Acético & $3 \%$ \\
Aditivo Control de Arcillas & $0,2 \%$ \\
Inhibidor de corrosión & $0,4 \%$ \\
Surfactante & $0.2 \%$ \\
\hline
\end{tabular}

La reacción del reservorio con el fluido diseñado muestra un incremento en la producción de agua y de petróleo, sin embargo, los resultados no son tan favorables, ya que existe un aumento de la producción de agua de aproximadamente del 24 $\%$, pero la producción de petróleo se llega a estabilizar favorablemente en comparación con el primer periodo $(\mathrm{m}=$ 0.63 y 1,28$)$.

El 8 de septiembre del 2017 y el 9 de febrero del 2018, se realizan una segunda y una tercera rehabilitación de pozos respectivamente para los cuales se utilizó un diseño de fluido de estimulación matricial ácida aumentando la cantidad del polímero para el control de arcillas (Composición de fluido II, y III).

En este segundo escenario, la producción de petróleo aumenta significativamente (1000 bbl/día), sin embargo, dura muy poco tiempo ya que se observa una declinación brusca $(\mathrm{m}=$ 7.73), por lo cual se llega a la conclusión de que el mecanismo de daño continúa presente en el reservorio luego de la estimulación matricial (migración de finos), (Halliburton, 2019).

Por lo que en el periodo de Agosto del 2018, se bombea nuevamente el mismo fluido de estimulación, pero esta vez enviando cantidades del fluido mayores, observando que la producción mantiene una declinación menor $(\mathrm{m}=0.07)$ durante aproximadamente 7 meses, sin embargo, la declinación incrementa conforme el corte de agua lo hace por lo que se evidencia que la migración de finos es un problema que se puede controlar durante un periodo de tiempo determinado, pero no se puede eliminar definitivamente (Civan, 2000), Figura 2.

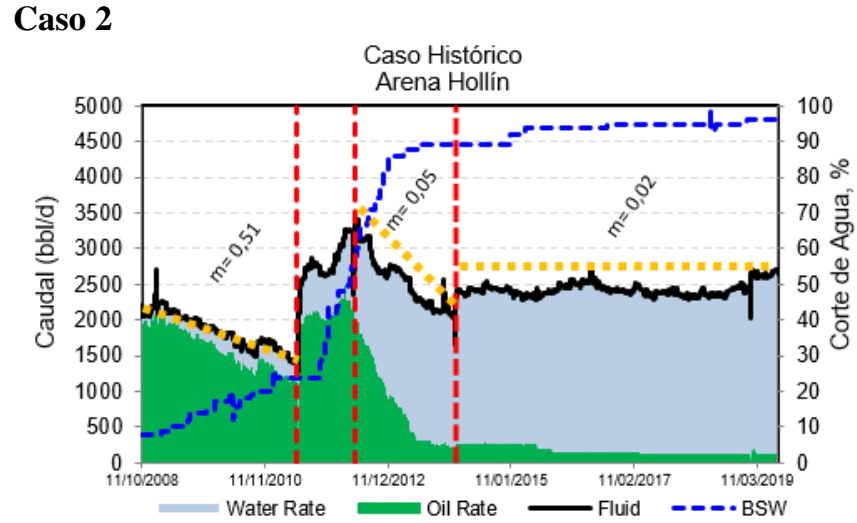

Figura 2. Caso 2 de Producción Campo de estudio

Este segundo caso muestra una situación diferente al previo, observa una declinación de la producción menor $(\mathrm{m}=0.51)$ en aproximadamente 2 años conforme aumenta el corte de agua, se procede a realizar un reacondicionamiento con el objetivo de restaurar la producción, sin embargo, al cabo de un año el corte de agua aumenta hasta un $90 \%$ por lo que la producción declina rápidamente.

A partir de este punto, observamos que la producción de fluido se mantiene constante, es decir no aumenta ni disminuye $(m=0.02)$ por lo que se puede concluir que ya han sido drenados todos los finos de migración; es decir ya no existe más arcillas que migrar a este punto.

Es importante recalcar que en este estado de producción ya es muy difícil realizar un tratamiento de remediación, es posible tratar de llegar a este estado en un periodo temprano con el objetivo de estabilizar la producción a través de los modificadores de permeabilidad relativa (Díaz et. al, 2009), sin embargo, toda acción de remediación se la debería realizar mucho antes.

\subsection{Geología}

El reservorio primario corresponde a una secuencia clástica de la formación cretácea Hollín (Leal, Gaibor, Garces, \& Benalcazar, 2015). La formación de Hollín incluye sedimentos fluviales marinos, dominados por mareas y poco profundos, que presentan diferentes características petrofísicas relacionadas con el entorno de deposición y diagénesis. La formación de Hollín se subdivide en dos miembros informales: Hollín Inferior y Hollín Superior.(Leal et al., 2015)

\subsubsection{Ambientes Sedimentarios}

Los ambientes de depositación son de vital importancia debido a que con ellos podemos conocer acerca de la sedimentología, depositación, facies y mineralogía de los diferentes reservorios de nuestro interés.

La formación Hollín se puede subdividir en dos miembros informales: Hollín Inferior y Hollín Superior. Sin embargo, desde la perspectiva sedimentológica, se han definido tres entornos de depósito: fluvial, mareal y estuarino (Rodríguez et al. 2014, Sandoval et al. (2015), y Leal et al. 2015), estos se describen a continuación: 


\subsubsection{Sistema Fluvial}

Depósitos Fluviales (también conocidos como Aluviales), abarca una amplia gama de sedimentos generados por la actividad de ríos, arroyos, y procesos de flujos asociados con la gravedad. Este tipo de sistemas ocurre en el presente bajo una variedad de condiciones climáticas y en diferentes ambientes tales como: desiertos, regiones húmedas y glaciales (Boggs, 2006).

\subsubsection{Sistema Estuarino}

Dalrymple, Zaitlin, and Boyd (1992) definen a un estuario como "La porción de mar adentro de un sistema de valles que reciben sedimentos de fuentes marinas como fluviales, en la cual contienen facies influenciadas por la marea, olas y procesos fluviales".

\subsubsection{Sistema Marino Somero}

Las líneas costeras no deltaicas reciben su suministro de sedimentos de una variedad de fuentes. Por ejemplo, los sedimentos pueden depositarse temporalmente en las desembocaduras de los ríos y luego ser recogidos y movidos a lo largo de la costa por olas y corrientes para formar playas. Además, los sedimentos pueden recogerse en el lecho marino y desplazarse hacia tierra, nuevamente para formar depósitos en la playa. En general, existe un transporte neto de arena en tierra, desde el fondo marino hasta la costa. Este transporte terrestre neto se produce debido al movimiento hacia el interior del agua por las olas generadas por el viento y las corrientes cercanas a la costa (Slatt, 2006).

\subsection{Verificación}

Al tener un indicio del problema se procedió a verificar la información antes analizada, para ello se tomó los datos arrojados de las pruebas de difracción de rayos $\mathrm{X}$ que mostraron la presencia de mineralogía específica, además se analizó las imágenes de láminas delgadas y por último se tomó los datos de un registro de rayos gamma espectral que sirvió para corroborar la presencia de ciertos minerales de manera cualitativa. Dentro de la base de datos se encontró una prueba de velocidad crítica que ayudó a entender el comportamiento de la producción con respecto a la permeabilidad.

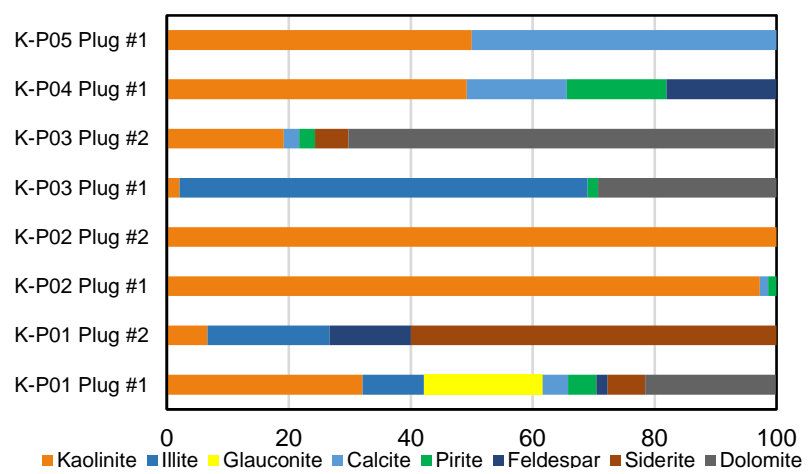

Figura 3. Mineralogía Arena Hollín de los principales campos de la Cuenca Oriente

Las principales arcillas que se encuentran en las diferentes muestras analizadas son Caolinita e illita, conocidas como arcillas de migración (intercambio de iones) las cuales son las causantes de perdida de permeabilidad del reservorio (Civan, 2000), lo que se ve reflejado en una disminución en la producción, Figura 3.

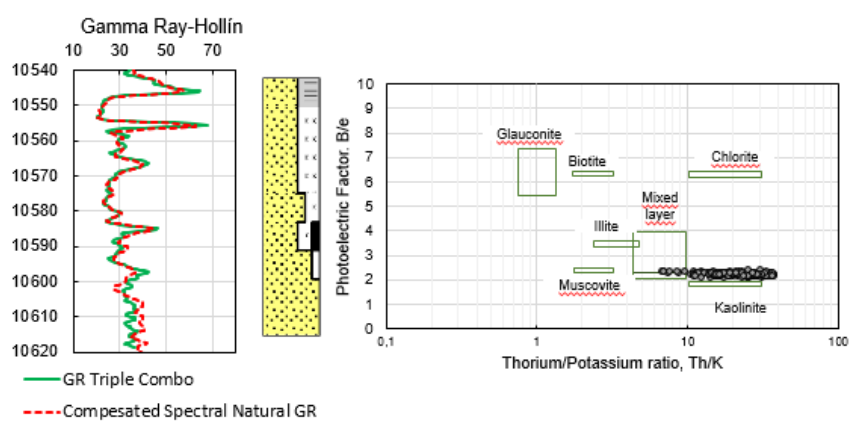

Figura 4. Rayos gamma, Registro litológico, y Análisis del Registro eléctrico para la arena Hollín del campo de estudio

Para la detección y comprobación de los minerales expuestos en las pruebas de difracción de rayos $\mathrm{X}$ se utilizó registros eléctricos, la curva de rayos gamma y los registros litológicos, Figura 4, se tomó datos de la curva de rayos gamma del campo de estudio para un ambiente fluvial de donde proviene la caolinita e illita (Slatt, 2006), el gráfico de factor fotoeléctrico vs la relación torio-potasio obtenido de los registros eléctricos del campo de estudio, muestra que el mineral que abunda en mayor cantidad es la caolinita.

\subsection{Análisis de posibles soluciones}

Una vez identificado el problema con el reservorio Hollín se procedió a recomendar tratamientos, para esto se tomó los datos de las diferentes pruebas de retorno de permeabilidad para sistemas ácidos, sistema de estabilización de finos, y modificadores de permeabilidad relativa, además se toman los datos de fluidos de control como método de prevención para la migración de finos.

\section{Pruebas de Retorno de Permeabilidad}

Una prueba de retorno de permeabilidad puede revelar daños en la formación, y se realiza utilizando un permeámetro de retorno. La porosidad y la permeabilidad de una muestra de núcleo se determinan haciendo fluir un aceite mineral refinado a través del núcleo. Para simular la invasión de fluido y filtrado en el núcleo, el fluido de reacondicionamiento se coloca contra el lado de salida del núcleo y la presión diferencial se aplica en la dirección opuesta a la de la medición de flujo anterior. Después de la contaminación, el aceite mineral fluye a través del núcleo en la dirección original y la porosidad resultante se compara con la porosidad original para determinar si se ha producido una reducción en la permeabilidad (Wilcox, Fisk, \& Corbett, 1987).

\section{RESULTADOS}

\subsection{Técnicas para la prevención en la migración de Finos}

\subsubsection{Fluidos de Control}

La pérdida de fluidos de terminación hacia el reservorio puede generar migración de finos si este no se formula con salinidad 
igual o mayor a la del yacimiento existirá intercambio iónico entre el fluido de control y el de reservorio provocando migración de finos durante las operaciones de terminación o reparación. Este fenómeno se observa en la Figura 5 donde los fluidos de control 6,7,8 y 9 no generan daño de formación y las demás formulaciones provocan una pérdida de permeabilidad de hasta el 8\% (Eoff \& Waltman, 2009).

Retorno de permeabilidad Vs Fluidos de Control

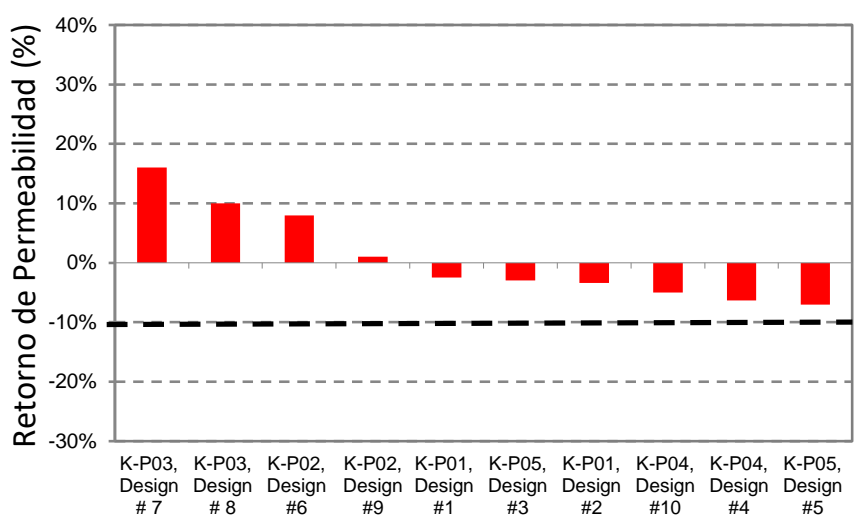

Figura 5. Resultados de pruebas de retorno de permeabilidad para fluidos de control

De acuerdo con los resultados obtenidos, existen retornos de permeabilidad del $15 \%$ y una pérdida de permeabilidad de hasta el $9 \%$ lo cual es aceptable para los diferentes diseños de fluidos de control.

Es importante mantener en observación las cantidades de aditivos que van a ser enviados con el fluido de control (solventes mutuales y surfactantes) ya que dosis inadecuadas, podrían desestabilizar el sistema (Economides \& Nolte, 2000) y provocar migración de finos.

\subsection{Técnicas para el control de Finos}

\subsubsection{Estimulación Ácida}

La estimulación ácida consiste en inyectar una solución ácida para disolver minerales como arcillas y feldespatos en la formación, que causan la migración de finos (Syed et al.,2016) , Figura 6.

Retorno de Permeabilidad Vs Fluidos Acidos

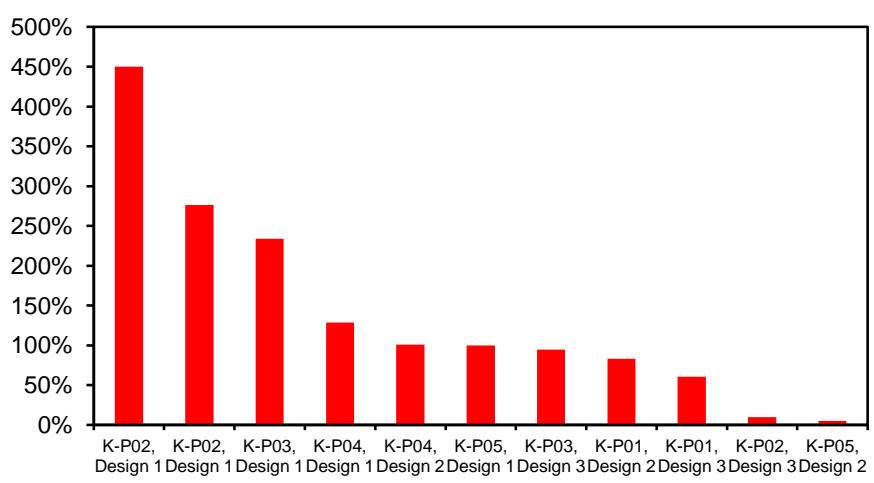

Figura 6. Resultados de pruebas de retorno de permeabilidad para sistemas ácidos
Para todas las pruebas realizadas se obtuvo resultados de retorno de permeabilidad positivos de hasta el $450 \%$ de retorno, con lo cual se comprueba que la estimulación ácida está totalmente recomendada para la arena Hollín.

\subsubsection{Fluido Estabilizador de Finos}

Los químicos utilizados para estabilizar las arcillas y los finos funcionan al ser adsorbidos, generalmente por atracción electrostática o intercambio iónico, en el reservorio de interés. Debido a que los silicatos tienen una carga negativa, el estabilizador más efectivo tiene una carga positiva (catiónica). Los estabilizadores de arcilla comunes son cationes altamente cargados, surfactantes cuaternarios, poliaminas, aminas policuaternarias y silanos orgánicos (Economides \& Nolte, 2000).

A velocidades de fluido suficientemente bajas las partículas finas permanecen unidas a la superficie del poro, cuando aumenta la velocidad del fluido existe una velocidad crítica donde comienza el desprendimiento de partículas por las fuerzas hidrodinámicas (Civan,2000), por lo tanto, la permeabilidad disminuye continuamente por la migración de finos que se acumulan.

Para demostrar el funcionamiento del tratamiento se realizó una prueba de velocidad crítica, Figura 7.

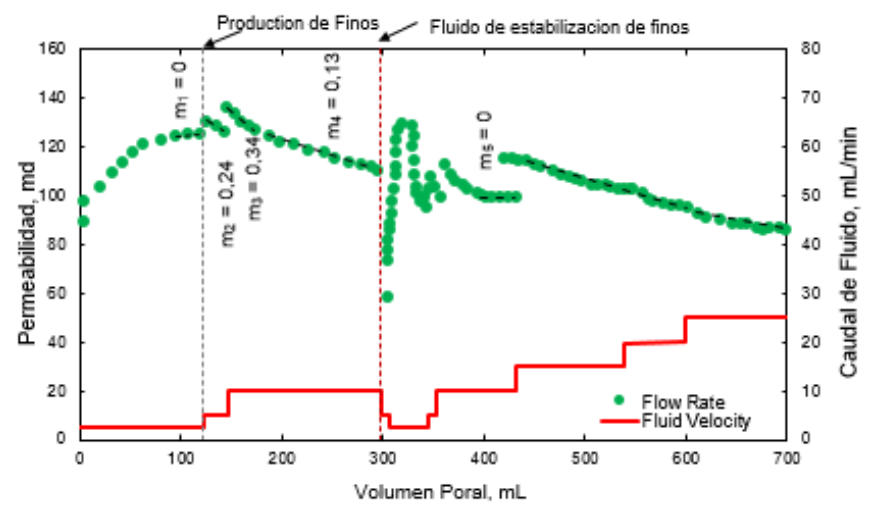

Figura 7. Prueba de velocidad crítica

Una vez que se determina la velocidad crítica de $10 \mathrm{ml} / \mathrm{min}$, se bombea el fluido estabilizador de finos y observamos una variación de la permeabilidad hasta que alcanza la velocidad de $10 \mathrm{ml} / \min (\mathrm{m}=0)$, donde observamos que la permeabilidad permanece constante, es decir no hay pérdida de permeabilidad, inclusive se logra aumentar la velocidad del fluido hasta $25 \mathrm{ml} / \mathrm{min}$ y no se observan cambios drásticos en la permeabilidad demostrando que el fluido estabilizador de finos funcionó.

Para un correcto diseño del tratamiento ácido se debe acondicionar la matriz con pre-flujos acuosos y ácidos con los que se evita que el ácido fluorhídrico tenga contacto con material calcáreo y el intercambio iónico con fluidos reservorio, luego bombea el sistema ácido principal para disolver los finos de migración, y finalmente se bombea el estabilizador de finos con el objetivo de que actúe en la cara de la formación y atrape aquellos finos que pudieran migrar en un futuro. 


\subsubsection{Modificadores de Permeabilidad relativa}

La fracción de agua es importante porque es directamente proporcional a la migración de finos (Civan,2000), con los RPM se busca alterar la curva de flujo fraccional. Si se atrapa una partícula de agua en la garganta poral, no permitirá que la partícula polar del aceite se adhiera al fino y la libere, por lo tanto, el sistema está incompleto para que se dé la migración de finos (García et al.,2008), Figura 8.

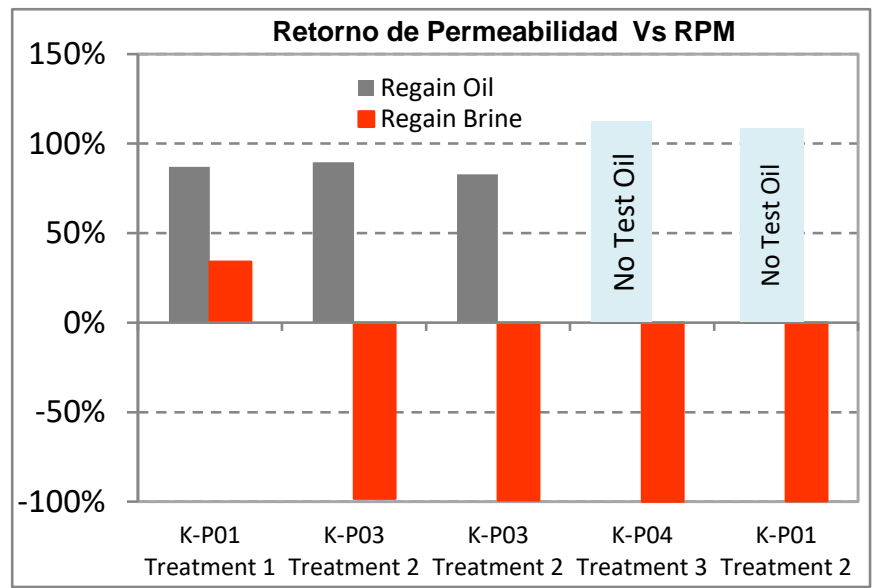

Figura 8. Resultados de pruebas de retorno de permeabilidad para modificadores de permeabilidad relativa

La acción de los modificadores de permeabilidad relativa los cuales buscan atenuar el incremento de corte de agua en el tiempo fue satisfactoria, ya que los resultados muestran un retorno permeabilidad de $0 \%$ al agua.

\subsubsection{Otros métodos: Fractura Hidráulica y métodos combinados.}

Cuando se fractura un pozo se tiene como objetivo disminuir el diferencial de presión $(\Delta \mathrm{P}=\operatorname{Pr}-\mathrm{Pwf})$, con ello se busca aumentar el índice de productividad, cada vez que se aumenta el índice de productividad, disminuye la velocidad intersticial del reservorio, entonces se produce más por debajo de la velocidad crítica. Entonces como se produce mucho más y el pozo está fracturado, no se tiene el término de turbulencia, debido a que la caída de presión y la velocidad crítica también disminuirán, si no se tiene turbulencia dentro del reservorio, los finos no migrarán taponando las gargantas porales (Economides \& Nolte,2000).

Si la velocidad intersticial es menor a la velocidad crítica no hay desprendimiento de finos (Civan,2000). El apuntalante brindará una mayor permeabilidad ya que este da la oportunidad de crear diámetros porales más amplios por donde los finos cruzarán sin quedarse atrapados y finalmente el uso del estabilizador de finos y los modificadores de permeabilidad relativa, combinados con la fractura crea una barrera adicional para la migración finos (Songire et al., 2014).

\subsection{Criterios de Selección}

Los criterios que se debe tomar en cuenta a la hora de seleccionar un tratamiento para el reservorio son: el índice de productividad, producción y tiempo, Figura 9.

La estimulación ácida da un IP alto, sin embargo la producción va a disminuir rápidamente por lo tanto el tiempo de intervenciones es constante; el estabilizador de finos da un IP bajo, sin embargo la producción no declina rápidamente y los tiempos de intervención del tratamiento son largos; los modificadores actúan de igual manera que los estabilizadores de finos sin embargo el tiempo de intervención es mucho más corto; y por último la fractura hidráulica y métodos combinados me ofrecen IP altos en ambos casos la diferencia radica en los tiempos de intervención, depende de lo que la empresa operada necesite.

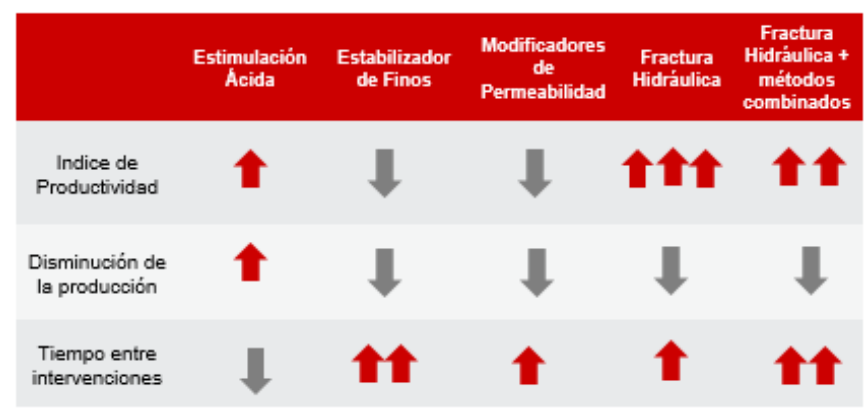

Figura 9. Criterio de Selección para los tratamientos de Control de Finos

\section{CONCLUSIONES}

El análisis mineralógico, ambiente depositacional y los registros eléctricos muestran al reservorio Hollín como una arena limpia, que eventualmente puede tener hasta un $40 \%$ de minerales distintos al cuarzo, entre ellos arcillas como caolinita e ilita.

La caolinita e illita (arcillas de migración), se desplazan al exponerse a fluidos de baja salinidad provocando un taponamiento de las gargantas porales lo cual provoca una disminución de permeabilidad en el reservorio.

Los casos históricos estudiados de producción y de velocidad crítica exponen un declive de producción conforme aumenta el corte de agua, debido a la diferencia de concentración de salinidad, provocando la migración de finos.

Entre los tratamientos químicos estudiados para mitigar el daño de formación por migración de finos se encuentran: estimulación ácida, agentes estabilizadores de finos, modificadores de permeabilidad relativa, fractura hidráulica y fracturamiento hidráulico combinado con agentes estabilizadores de finos.

En el caso de las pruebas de retorno de permeabilidad para fluidos ácidos se observa un efecto positivo en los diferentes escenarios, ya que se conserva la permeabilidad hasta un $400 \%$; sin embargo, este tratamiento se debe mantener durante la vida productiva del pozo para que los niveles de producción sean rentables. 
La acción de los modificadores de permeabilidad relativa los cuales buscan atenuar el incremento de corte de agua en el tiempo fue satisfactoria ya que los resultados muestran una permeabilidad reganada de $0 \%$ al agua.

Con el tratamiento de estabilización de finos la declinación en la producción disminuye, y el tiempo de acción es duradero, sin embargo, el índice de productividad también se ve afectado.

El fracturamiento hidráulico es uno de los métodos en los que tenemos índices de productividad, y tiempos de duración muy altos que nos proporcionan una extensa recuperación en producción.

Al combinar fractura hidráulica con agentes estabilizadores de finos, se incrementa el índice de productividad y se logra prolongar la vida productiva del pozo.

\section{REFERENCIAS}

Ahmed, T. (2001). Reservoir Engineering Handbook. In Reservoir Engineering Handbook (2nd ed.). Houston: Texas. 70-75, 130, 245-250, $266,275$.

Atahualpa, G., \& Trujillo, B. (2013). Efecto de la Glauconita en las propiedades petrofísicas del reservorio arenisca " $T$ " superior del bloque Tarapoa. Universidad Central del Ecuador. Quito: Ecuador, 70-80 Obtenido de: file:///C:/Users/Usuario/Downloads/T-UCE-0012156.pdf

Baby, P., Rivadeneira, M., \& Barragán, R. (2014). La Cuenca Oriente: Geología Y Petróleo. (3era ed.), 415.

Boggs, S. (2006). Principles of sedimentology and Stratigraphy. New Jersey: Estados Unidos, 300.

Boyd, R., Dalrymple, R.W. \& Zaitlin, B.A. (1992) Classification of clastic coastal depositional environments. Sedimentary Geology, 80, 139-150. https://doi.org/10.1016/0037-0738(92)90037-R

Catuneanu, O. (2006). Principles of Sequence Stratigraphy (1era ed.). Canada: Alberta. 15-17, 156

Chafetz, H. S., \& Reid, A. (2017). Syndepositional shallow-water precipitation of glauconitic minerals Syndepositional shallow-water precipitation of glauconitic minerals. 38-39. https://doi.org/10.1016/S0037-0738(00)00082-8

Civan, F. (2000). Reservoir Formation Damage. (1era ed.). Houston: Texas Gulf Publishing Company. 150-155, 232-235, 255-266.

Danna, J. (2006). Manual de Mineralogía. (2da ed.), 2, 30-48. España: Madric Obtenido de: https://www.studocu.com/ec/document/universidadcatolica-del-norte/mineralogia/otros/manual-de-mineralogia-danahurlbut-2a-edicion-geo-libros-pdf/5114231/view

Dalrymple, R.W., Zaitlin, B.A. \& Boyd, R. (1992) Estuarine facies models conceptual basis and stratigraphic implications. Journal of Sedimentary Petrology, 62, 1130-1146.

Diaz, G., Castillo, P., Villa, K., Sanchez, L., Mucci, M., Robles, J., ... Peacock, H. (2009). Fracture conformance treatments using RPM: Efficiency and durability evaluation. SPE Latin American and Caribbean Petroleum Engineering Conference Proceedings, 3, 12791289.

https://doi.org/10.2118/122913-MS

Economides, M. J., \& Nolte, K. G. (2000). Reservoir Stimulation (3era ed.; E. Kapitan, Ed.). Texas: Jonh Wiley \& Sons Ltd.
Eoff, L., \& Waltman, B. (2009). Polymer Treatment Controls Fluid Loss While Maintaining Hydrocarbon Flow. JPT, 61(7), 3. https://doi.org/10.2118/0709-0028-JPT

Freed, R. (1976). Clay Diagenesis and Abnormally High Fluid Pressure. 4-6 SEG-1982-0505

Garcia, B., Soriano, J. E., Chacon, W., \& Eoff, L. S. (2008). Novel AcidDiversion Technique Increases Production in the Cantarell Field, Offshore Mexico. 1-15. https://doi.org/10.2118/112413-ms

Halliburton. (2019). Production Enhancement Ecuador Stimulation Handbook (p. 450). p. 450. Ecuador.

Johnson, C. L., Stright, L., Purcell, R., \& Durkin, P. (2017). Stratigraphic evolution of an estuarine fill succession and the reservoir characterization of inclined heterolithic strata, Cretaceous of southern Utah, USA. Geological Society, London, Special Publications, 444(1), 251-286. https://doi.org/10.1144/SP444.1

Leal, J., Gaibor, J., Garces, S., \& Benalcazar, E. (2015). New Approaching for Rock Types and Lithofacies Synergy for the Hollin Formation, Palo Azul Field, Ecuador. 2-3. https://doi.org/10.2118/177156-ms

Morrow, D. W. (1982). Diagenesis 1. Dolomite - Part 1: The Chemistry of Dolomitization and Dolomite Precipitation. Geoscience Canada, 9(1). Obtenido de: https://journals.lib.unb.ca/index.php/GC/article/view/3279

Reinoso, W., Torres, F., Aldana, M., Campo, P., Alvarez, E., \& Tovar, E. (2016). Removing Formation Damage From Fines Migration in the Putumayo Basin in Colombia: Challenges, Results, Lessons Learned, and New Opportunities after More Than 100 Sandstone Acidizing Treatments. Society of Petroleum Engineers. doi:10.2118/178996-MS

Rodriguez, M., Abad, F., Rodriguez, L., Gaibor, J., Moran, M., \& Verdezoto, A. (2015). Application of Modern Reservoir Characterization in Mature Fields to Unravel Hidden Reserves. Society of Petroleum Engineers. https://doi.org/10.2118/177195-ms

Sandoval, J., Gaibor, J., Benalcazar, E., \& Jimenez, L. (2015). State of the Art in 3D Reservoir Characterization: Palo Azul Field, Ecuador. Society of Petroleum Engineers. 18-20. https://doi.org/10.2118/177148-ms

Schechter, R. S. (1992). Oil well stimulation improved. In Hart's E and P: New Jersey. 125-130, 136

Slatt, R. M. (2006). Stratigraphic Reservoir Characterization for Petroleum Geologists, Geophysicists and Engineers. (J. Cubitt, Ed.). Obtenido de: www.academia.edu

Songire, S., Uppuluri, R., \& Rama, M. S. (2014). Aqueous-Based Surface Modifying Agent for Mitigation of Fines Migration. Society of Petroleum Engineers, 12-14. https://doi.org/10.2118/172273-MS

Syed, A., Kalfayan, L., \& Montmogomery, C. (2016). Acid Stimulation (26ava ed.). Society of Petroleum Engineers.

Thompson, G. R., \& Hower, J. (1975). The Mineralogy Of Glauconite. 23, 289-300. Great Britain. Obtenido de: http://www.clays.org/journal/archive/volume\%2023/23-4-289.pdf

Triplehorn, D. M. (1965). Origin and Significance of Glauconite in the Geologic Sequence: Abstract. 33, 282-283. Obtenido de: http://archives.datapages.com/data/tgs/digest/data/033/033001/282_t gs330282a.htm

Vasquez, J., Waltman, B., \& Eoff, L. (2010). Field implementation of a nove solids-free system to minimize fluid loss during overbalanced workover operations. Society of Petroleum Engineers. https://doi.org/10.2118/130210-MS

Wilcox, R. D., Fisk, J. V., \& Corbett, G. E. (1987). Filtration Method Characterizes Dispersive Properties of Shales. SPE Drilling Engineering, 2(02), 149-158. https://doi.org/10.2118/13162-PA 


\section{BIOGRAFÍAS}

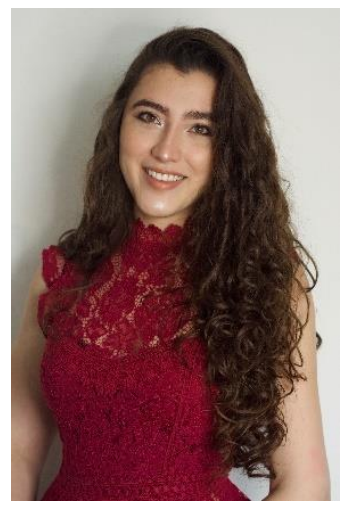

Mayra Jizbell Ruilova Zambrano, Ingeniera de Petróleos de la Universidad Central del Ecuador (2019). Realizó prácticas preprofesionales en diversas empresas como Halliburton (2018) Y Repsol Ecuador S.A (2019). Colaboró en un proyecto interno para el Departamento de Desarrollo de Yacimientos de la compañía Repsol S.A. (2019). Participó como expositor en el evento Next Wave Paper Contest 2019 de la SPE Sección Ecuador siendo acreedora del segundo lugar. Actualmente se desempeña como ingeniera en entrenamiento para Schlumberger.

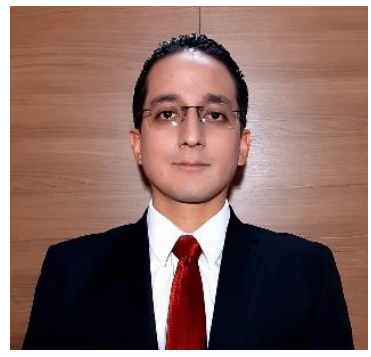

Álvaro Javier Izurieta

Ballesteros, Ingeniero en Petróleos, Escuela Politécnica Nacional (2010), Especialista Industria Petroquímica, Universidad Nacional General San Martin (2017) se desempeña actualmente como Asesor Técnico para Halliburton. Sus intereses son la optimización de producción mediante fracturamiento hidráulico, estimulación acida, ingeniería de producción de petróleo e ingeniería de producción. Es autor y coautor de varios estudios técnicos para la Sociedad de Ingenieros Petroleros (SPE) y Congreso Mexicano del Petróleo (CMP). 